than obstruction and, therefore, in search of an explanation, the authors focus on factors affecting lung size. Here they suggest the 'Barker hypothesis' of poor fetal growth as a possible common causal factor responsible for small lungs, cardiovascular disease, diabetes and other chronic conditions. ${ }^{14}$ Although this theory cannot be proved in an isolated study of an adult population, their suggestions are in line with studies showing strong 'tracking' characteristics of lung function. ${ }^{15}$ However, other explanations are also possible, such as sharing of common genes affecting handling of oxidative stress or genes responsible for detoxification or tissue repair mechanisms.

Poor fetal growth was undoubtedly present at the time of Hutchinson, although the spectrum of pulmonary diseases at that time differed greatly from what we see today in Western countries. While tuberculosis and other lung infections were the main pulmonary killers at that time, diseases such as COPD and lung cancer were rare. Yet, it is amazing that the predictive power of a simple measurement of expired air is still as strong today as it was $>150$ years ago. Unfortunately, as described by Petty in his brilliant editorial on Hutchinson and his mysterious machine, the spirometer never reached a similar popularity to the sphygmomanometer, which was invented $\sim 50$ years later. ${ }^{16}$ This is probably the main reason why the patients of today seldom spontaneously ask their doctors for a measurement of lung capacity, whereas they often wish to have their blood pressure measured. This leads to the typical situation, whereby many patients have their first spirometry performed 10-20 years too late, and also results in the frustrating observation that in many of them, more than half of their lung capacity has already been lost! The fact that we today, $>150$ years after the invention of the spirometer, still have problems explaining why VC is so vital for future health should, however, not discourage us-studies such as that of Burney and Hooper underline that spirometry should be a part of every standard medical assessment just like the measurement of blood pressure.

\section{Competing interests None.}

Provenance and peer review Commissioned; not externally peer reviewed.

Published Online First 26 October 2010

Thorax 2011;66:1-2. doi:10.1136/thx.2010.151274

\section{REFERENCES}

1. Hutchinson J. On the capacity of the lungs, and on respiratory function, with a view of establishing a precise and easy method of detecting disease by spirometer. Med Chir Trans (Lond) 1846:29:137-61.

2. Peabody FW, Wentworth J. Clinical studies of respiration. The vital capacity of the lungs and its relation to dyspnea. Arch Intern Med 1917;20:443-67.

3. Ashley F, Kannel WB, Sorlie, et al. Pulmonary function: relation to ageing, cigarette habit, and mortality. The Framingham Study. Ann Intern Med 1975:82:739-45.
4. Friedman GD, Klatsky AL, Siegelaub AB. Lung function and risk of myocardial infarction and sudden cardiac death. N Engl J Med 1976;294:1071-5.

5. Cohen BH. Is pulmonary disfunction the common denominator for the multiple effects of cigarette smoking? Lancet 1978;2:1024-27.

6. Lange $\mathbf{P}$, Nyboe J, Appleyard M, et al. Spirometric findings and mortality in never-smokers. J Clin Epidemiol 1990;43:867-73.

7. Sorlie PD, Kannel WB, O'Connor G. Mortality associated with respiratory function and symptoms in advanced age. Am Rev Respir Dis 1989; 140:379-84

8. Eisen EA, Dockery DW, Speizer FE, et al. The association between health status and the performance of excessively variable spirometry test in a population study in six U.S. cities. Am Rev Respir Dis 1987:136:1371-76.

9. Sin D, Man SFP. Why are patients with chronic obstructive pulmonary disease at increased risk of cardiovascular diseases? Circulation 2003;107:1514-19.

10. Mannino DM, Holguin F, Pavlin Bl, et al. Risk factors for prevalence of and mortality related to restriction. Int J Tuberc Lung Dis 2005;9:613-21.

11. Burney PGJ, Hooper R. Forced vital capacity, airway obstruction and survival in a general population sample from the USA. Thorax 2011;66:49-54.

12. Celli B, Cote C, Marin J, et al. The body-mass index airflow obstruction, dyspnea, and excercice capacity index in chronic obstructive pulmonary disease. N Engl J Med 2004;350:1005-12.

13. Puhan M, Garcia-Aymerich J, Frey $\mathbf{M}$, et al. Expansion of the prognostic assessment of patients with chronic obstructive pulmonary disease: The updated BODE index and the ADO index. Lancet 2009;374:704-11.

14. Barker DJP, Bull AR, Osmaond C, et al. Fetal and placental size and risk of hypertention in adult life. BMJ 1990;301:259-62.

15. Hancox RJ, Poulton R, Greene JM, et al. Associations between birth weight, early childhood weight gain and adult lung function. Thorax 2009;64:228-32.

16. Petty TL. John Hutchinson's mysterious machine revisited. Chest 2002;121:219S-23.

\title{
Echocardiography, troponins and lower extremity ultrasound: the 'Three Musketeers' lead the prognosis of acute pulmonary embolism
}

\section{Antonio Vitarelli}

The European guidelines ${ }^{1}$ and American guidelines ${ }^{2}$ highlight that, in the diagnosis

Sapienza University, Cardio-Respiratory Department, Rome, Italy

Correspondence to Professor Antonio Vitarelli, Via Lima 35, 00198 Rome, Italy; vitar@tiscali.it and management of acute pulmonary embolism (PE), the functional consequences determined by right ventricular (RV) dysfunction and elevation of cardiac biomarkers are more relevant for risk stratification than assessment of the anatomical burden and distribution of the pulmonary artery thrombus. The mortality rate associated with massive $\mathrm{PE}$ may reach $30 \%$, while that associated with so-called submassive PE (defined as the presence of RV dysfunction without systemic hypotension) is between $5 \%$ and $10 \%$ and that associated with non-massive $\mathrm{PE}$ is $<5 \%$. ${ }^{1}$ While there is consensus that thrombolytic therapy, catheter embolectomy or surgery are indicated in patients with right heart failure and haemodynamic instability, the appropriate treatment of patients with submassive $\mathrm{PE}$ remains controversial. In this subset of patients, the "tricks of the trade' ${ }^{3}$ should be identified and clinical-laboratory aspects evaluated to judge the level of severity. RV echocardiographic parameters, cardiac troponins and peripheral ultrasound data are described as poor prognostic factors in the currently available literature.

Each of these tests has its own advantages and limitations. A number of studies have shown that RV dysfunction and 
dilation is a robust prognostic factor in acute PE. Still, some questions arise. First, the different pathophysiology of acute versus chronic pulmonary hypertension has recently been outlined. ${ }^{4}$ Similarities between PE (acute occlusive pulmonary hypertension) and chronic pulmonary hypertension include the fact that pulmonary circulatory resistance increases, RV function may decline and mortality and morbidity are correlated with the extent of RV dysfunction in both cases. Nevertheless, the mechanisms of cardiopulmonary changes are very different and result in different modes of $\mathrm{RV}$ injury that require unique therapeutic targets. Second, no uniform criteria have been used in different studies to assess the presence of RV dysfunction. ${ }^{5}{ }^{6}$ In the majority of studies, RV dysfunction was defined as RV hypokinesis as assessed by qualitative evaluation of RV wall motion. The quantitative assessment has included RV dilation (end-diastolic diameter $>30 \mathrm{~mm}$ in parasternal view, RV-LV end-diastolic diameter ratio $>1$ in fourchamber view, RV-LV end-diastolic diameter ratio $>0.6$ in parasternal or subcostal views, RV end-diastolic area $>20 \mathrm{~cm}^{2}$ in apical, subcostal or transoesophageal fourchamber views), RV wall hypertrophy (free wall thickness $>5 \mathrm{~mm}$ in parasternal or subcostal views), dilation of the right pulmonary artery $\left(>12 \mathrm{~mm} / \mathrm{m}^{2}\right)$, loss of inspiratory collapse of the inferior vena cava and pulmonary hypertension (defined as Doppler pulmonary acceleration time $<90 \mathrm{~ms}$ or the presence of a right ventricular-atrial gradient $>30 \mathrm{~mm} \mathrm{Hg}$ ). Lastly, new echocardiographic parameters of RV dysfunction are being studied ${ }^{7}$ and, with recent advances in Doppler and tissue Doppler echocardiography, new methods for measuring regional and global RV function or contractility have been suggested. ${ }^{8}$ Thus, a uniformly accepted definition of the criteria for echocardiographically-detected $\mathrm{RV}$ dysfunction would be desirable to give a conclusive answer on the prognostic significance of echocardiographic RV dysfunction in haemodynamically stable patients with PE.

Several studies have attempted to identify laboratory data which have prognostic power in patients with acute PE. Many of these have focused on cardiac biomarkers including the troponins and natriuretic peptides which correlate with RV dysfunction on echocardiography. Comprehensive meta-analyses of the prognostic value of troponins in acute PE have been reported. ${ }^{9}$

10 Their prognostic value was consistent among the studies, regardless of the specific assay and the relative cut-off point. The results were consistent for both troponin I and $\mathrm{T}$. However, it is unclear whether thrombolysis has a role in the management of haemodynamically stable patients on the basis of troponin elevation and, if it has, which patients should be selected for this treatment.

The prognosis in the presence of lower limb deep vein thrombosis (DVT) evaluated with compression ultrasound (CUS) in patients with symptomatic PE has been investigated. ${ }^{11}{ }^{12}$ Multivariate analysis showed that active cancer, inadequate anticoagulation, leg symptoms, male gender, presence of DVT, presence of proximal DVT and previous DVT were independent risk factors for an adverse outcome. Whole leg CUS, which captures images from the iliac to the calf veins, may improve initial detection of distal DVT. Bilateral examination is justified especially in patients with a history of an active malignancy because of the high incidence of asymptomatic contralateral thrombosis. However, there is still much debate about the importance of DVT as a significant risk factor for the onset of PE. While several investigators have reported the important role of lower extremity CUS in the follow-up of PE, others found no significant difference in the risk of recurrent thromboembolic events or death among patients with PE with and without DVT. ${ }^{13}$ Each patient might have different risks for thrombosis or bleeding and potentially adverse consequences despite prophylaxis. What is best epidemiologically for the group is not necessarily what is best clinically for the individual patient.

Jiménez et al assessed the ability of three diagnostic tests (cardiac troponin I, echocardiography and lower extremity CUS) obtained soon after diagnosis of PE to prognosticate the primary study outcome-PE-related death-during 30 days of follow-up after PE diagnosis. ${ }^{14}$ Multivariate logistic regression confirmed these tests as significant predictors of death from PE. Use of any two-test strategy had a higher specificity and positive predictive value than either test alone. They concluded that, in haemodynamically stable patients with $\mathrm{PE}$, a combination of echocardiography (or troponin testing if echocardiography is not readily available) and CUS improved prognostication compared with the use of each test alone for identification of those at high risk for PE-related death.

Previous studies have been published in this area ${ }^{15-18}$ but have provided equivocal findings regarding performance of test combinations for prognosticating PE-related adverse events. Whereas in some reports the addition of echocardiographic data to the blood test data did not improve the test characteristics, ${ }^{15}$ in others the combination of troponin I and echocardiography improved the prognostic value compared with each test alone. $^{16-18}$ The paper by Jiménez and colleagues adds to the existing literature by providing a valuable contribution to this important debate and by showing that, among the two-test strategies, the combination of echocardiography and venous ultrasound had the best test characteristics of positive predictive value and positive likelihood ratio in all study patients and in the high-risk subgroup. This study supplies useful insights into risk stratification in normotensive patients presenting with acute $\mathrm{PE}$, since there is a growing interest in the early discharge and outpatient management of subjects presenting with acute PE who are haemodynamically stable. Owing to the high rate of hospital mortality in this group, it is important that every attempt is made to identify those at the highest risk who could not be treated in an outpatient setting and could require close monitoring or even more aggressive therapy.

Some cautions should be noted in evaluating the conclusions drawn from this study. The particular approach may depend on available hospital resources, especially whether echocardiography can be readily obtained on holidays. Echocardiographic images are useful only if the $\mathrm{RV}$ free wall can be clearly defined. In any case, assessment of RV function can also be challenging with good acoustic windows and even with other alternative techniques such as CT scanning. ${ }^{19}$ In the presence of persistent elevation of troponins and the absence of coronary angiography data, a significant occlusion of coronary vessels cannot be excluded, especially in patients with ST segment elevation. Besides troponins, increased B-type natriuretic peptides were also found to be an independent prognostic marker of morbidity and mortality in acute PE. ${ }^{10} 16$ Patients with increased levels of both natriuretic peptides and troponins are at a particularly high risk of adverse outcomes, and natriuretic peptides appear to be especially helpful in identifying lowrisk patients, given their very low positive rate among those with a poor outcome. The relative contribution of troponins and natriuretic peptides for risk stratification in clinical practice remains to be defined. 
However, undoubtedly the use of echocardiography (including transoesophageal echocardiography), laboratory findings and venous ultrasonography should be encouraged in patients with suspected high-risk PE. Management decisions should be taken based on all collected data on a case by case basis. Each test comes to the rescue of the others. 'One for all and all for one'.

\section{Competing interests None.}

Provenance and peer review Commissioned; not externally peer reviewed.

Published Online First 12 November 2010

Thorax 2011;66:2-4. doi:10.1136/thx.2010.151316

\section{REFERENCES}

1. Torbicki A, Perrier A, Konstantinides S, et al; Task Force for the Diagnosis and Management of Acute Pulmonary Embolism of the European Society of Cardiology. Guidelines on the diagnosis and management of acute pulmonary embolism. Eur Heart J 2008;29:2276-315

2. Kearon C, Kahn SR, Agnelli G, et al; American College of Chest Physicians. Antithrombotic therapy for venous thromboembolic disease: American College of Chest Physicians Evidence-Based Clinical Practice Guidelines (8th Edition). Chest 2008;133(6 Suppl):454S-545S.
3. Goldhaber SZ. Assessing the prognosis of acute pulmonary embolism: tricks of the trade. Chest 2008;133:334-6.

4. Watts JA, Marchick MR, Kline JA. Right ventricular heart failure from pulmonary embolism: key distinctions from chronic pulmonary hypertension. J Card Fail 2010;16:250-9.

5. Toosi MS, Merlino JD, Leeper KV. Prognostic value of the shock index along with transthoracic echocardiography in risk stratification of patients with acute pulmonary embolism. Am J Cardiol 2008;101:700-5.

6. Sanchez 0, Trinquart L, Colombet I, et al. Prognostic value of right ventricular dysfunction in patients with haemodynamically stable pulmonary embolism: a systematic review. Eur Heart J 2008;29:1569-77.

7. Holley AB, Cheatham JG, Jackson JL, et al. Novel quantitative echocardiographic parameters in acute PE. J Thromb Thrombolysis 2009;28:506-12.

8. Vitarelli A, Terzano C. Do we have two hearts? New insights in right ventricular function supported by myocardial imaging echocardiography. Heart Fail Rev 2010;15:39-61.

9. Becattini C, Vedovati MC, Agnelli G. Prognostic value of troponins in acute pulmonary embolism: a meta-analysis. Circulation 2007;116:427-33.

10. Lega J-C, Lacasse $Y$, Lakhal L, et al. Natriuretic peptides and troponins in pulmonary embolism: a meta-analysis. Thorax 2009;64:869-75.

11. Jiménez D, Aujesky D, Díaz G, et al; the RIETE Investigators. Prognostic significance of concomitant deep vein thrombosis in patients presenting with acute symptomatic pulmonary embolism. Am J Respir Crit Care Med 2010;181:983-91.
12. Yamaki T, Nozaki M, Sakurai H, et al. Presence of lower limb deep vein thrombosis and prognosis in patients with symptomatic pulmonary embolism: preliminary report. Eur J Vasc Endovasc Surg 2009;37:225-31.

13. Girard P, Sanchez 0, Leroyer C, et al. Deep venous thrombosis in patients with acute pulmonary embolism: prevalence, risk factors, and clinical significance. Chest 2005;128:1593-600.

14. Jiménez D, Aujesky D, Moores L, et al. Combinations of prognostic tools for identification of high-risk normotensive patients with acute symptomatic pulmonary embolism. Thorax 2011;66: 75-81.

15. Kostrubiec M, Pruszczyk P, Bochowicz A, et al. Biomarker-based risk assessment model in acute pulmonary embolism. Eur Heart J 2005;26:2166-72.

16. Binder L, Pieske B, Olschewski $\mathrm{M}$, et al. N-terminal pro-brain natriuretic peptide or troponin testing followed by echocardiography for risk stratification of acute pulmonary embolism. Circulation 2005;112:1573-9.

17. Scridon T, Scridon C, Skali H, et al. Prognostic significance of troponin elevation and right ventricular enlargement in acute pulmonary embolism. $\mathrm{Am} \mathrm{J}$ Cardiol 2005:96:303-5.

18. Stein PD, Matta F, Janjua M, et al. Outcome in stable patients with acute pulmonary embolism who had right ventricular enlargement and/or elevated levels of troponin I. Am J Cardiol 2010;106:558-63.

19. Vorobiof G, Kadiev S. Evaluation of right ventricular size on computed tomography: unreliable at best. Am J Med 2008;121:e15; author reply e17.

\section{How does diesel exhaust impact asthma?}

\section{John R Balmes}

Both air pollution health effects researchers and air quality regulatory agencies have been paying increased attention to emissions from motor vehicles in recent years. A growing body of scientific literature supports the concept that exposure to roadways with high traffic density is associated with adverse health effects, including increased risk of negative asthma outcomes. Heavy-duty diesel-powered vehicles like trucks and buses are often driven more frequently on roadways with high traffic density and, as such, diesel exhaust has been suspected to be a major cause of traffic-associated asthma morbidity.

University of California, San Francisco and Berkeley, USA Correspondence to John R Balmes, University of California, San Francisco and Berkeley, USA; jbalmes@medsfgh.ucsf.edu
Diesel exhaust is somewhat akin to tobacco smoke in that it is a mixture of particles and gases with many chemical constituents. Diesel exhaust particulate (DEP) is mostly elemental carbon with about 20-40\% adsorbed organic compounds, but sulfates, nitrates and metals are also present. ${ }^{1}$ Polycyclic aromatic hydrocarbons (PAHs) and related compounds such as quinones have been touted as the most toxicologically relevant constituents of DEP, primarily because of their redox potential and ability to cause oxidative stress. ${ }^{2}$ More than $90 \%$ of DEP mass is in particles $>1 \mu \mathrm{m}$ in diameter that can easily be inhaled into the deep lung. ${ }^{1}$ The vapour phase of diesel exhaust includes carbon monoxide, oxides of nitrogen, sulfur oxides and volatile organic compounds, many of which are known to be respiratory tract irritants such as formaldehyde, acrolein and naphthalene (a volatile PAH).
Many in vitro and animal experimental studies support the toxicity of DEP. ${ }^{1-4}$ The concept of a tiered response to DEP that is dose-dependent has been advanced which posits that low doses induce oxidative stress and upregulation of antioxidant and phase II enzymes, intermediate doses lead to activation of inflammatory signalling cascades and higher doses to cytotoxicity and apoptosis. $^{2}$ Organic extracts of DEP have been studied with regard to their ability to induce oxidative stress and the polar fraction containing quinones showed the greatest effect. ${ }^{2}$ Mechanisms by which DEP could potentially exacerbate asthma include enhancement of airway inflammation, non-specific airway hyperresponsiveness and specific allergic responses. Several groups have shown that DEP can act as an adjuvant when combined with an experimental allergen resulting in enhanced IgE antibody production and increased allergic inflammation and airway hyper-responsiveness in mice. ${ }^{56}$ One such study showed that treatment with the antioxidant $\mathrm{N}$-acetyl cysteine blunted the adjuvant effects of DEP, providing further supportive evidence for the role of oxidative stress in DEP toxicity. ${ }^{6}$

A number of studies have found increased risks of asthma outcomes in 